\title{
A DEEP LOOK AT THE T-TYPE BROWN DWARF BINARY $\epsilon$ INDI Bab WITH CHANDRA AND THE AUSTRALIA TELESCOPE COMPACT ARRAY
}

\author{
M. Audard, ${ }^{1}$ A. Brown, ${ }^{2}$ K. R. Briggs, ${ }^{3}$ M. Güdel, ${ }^{3}$ A. Telleschi, ${ }^{3}$ And J. E. Gizis ${ }^{4}$ \\ Received 2005 March 4; accepted 2005 April 12; published 2005 May 2
}

\begin{abstract}
We present deep observations of the nearby T-type brown dwarf binary $\epsilon$ Indi Bab in radio with the Australia Telescope Compact Array and in X-rays with the Chandra X-Ray Observatory. Despite long integration times, the binary (composed of T1 and T6 dwarfs) was not detected in either wavelength regime. We reached $3 \sigma$ upper limits of $1.23 \times 10^{12}$ and $1.74 \times 10^{12} \mathrm{ergs} \mathrm{s}^{-1} \mathrm{~Hz}^{-1}$ for the radio luminosity at 4.8 and $8.64 \mathrm{GHz}$, respectively; in X-rays, the upper limit in the $0.1-10 \mathrm{keV}$ band was $3.16 \times 10^{23} \mathrm{ergs} \mathrm{s}^{-1}$. We discuss the above results in the framework of magnetic activity in ultracool, low-mass dwarfs.
\end{abstract}

Subject headings: radio continuum: stars — stars: activity — stars: coronae — stars: individual ( $\epsilon$ Indi Bab) stars: low-mass, brown dwarfs - X-rays: stars

\section{INTRODUCTION}

Magnetic activity is a common feature of late-type stars. The general hypothesis assumes that some magnetic dynamo mechanism $(\alpha-\omega$ dynamo) occurs at the interface between the radiative core of the star and its outer convective zone. However, objects with masses below $0.3 M_{\odot}$ (spectral type later than M3) are generally believed to be fully convective and thus cannot support the $\alpha-\omega$ dynamo. Nevertheless, magnetic activity is commonly seen, even in very late M-type objects (e.g., Johnson 1981, 1987; Tagliaferri et al. 1990; Drake et al. 1996; Fleming et al. 2000; Rutledge et al. 2000; Fleming et al. 1993, 2003; Stelzer 2004). An alternative dynamo mechanism ( $\alpha^{2}$ dynamo), involving turbulent magnetic fields, has been proposed to take place in fully convective objects (e.g., Durney et al. 1993).

Studies of the $\mathrm{H} \alpha$ emission (a common tracer of chromospheric activity) in late-type stars have shown a steep decrease for spectral types later than M7 despite their fast rotation (Gizis et al. 2000; Mohanty \& Basri 2003). The X-ray emission also appears to decrease, although the sample of studied stars is limited (e.g., Fleming et al. 1993, 2003). It was thus a surprise when Berger (2002) found no decline in radio activity between spectral types M3 and L3.5, and even suggested a possible increase in radio luminosity with cooler effective temperature. Recent results seem to support such a trend in late M/early L dwarfs (Berger et al. 2005; Burgasser \& Putnam 2005). Old T-type brown dwarfs are, therefore, attractive objects to observe, in order to understand magnetic activity in ultracool dwarfs. However, observations of $\mathrm{T}$ dwarfs are scarce in the radio and were nonexistent in X-rays.

This Letter extends the previous investigations into the $\mathrm{T}$ dwarf domain. We present the first observation of a $T$ dwarf in X-rays with the Chandra X-Ray Observatory, along with a deep observation in the radio regime with the Australia Telescope Compact Array (ATCA). Our target, $\epsilon$ Ind Bab, is the closest known brown dwarf and is composed of two objects with spectral types $\mathrm{T} 1$ and $\mathrm{T} 6$ separated by $00^{\prime \prime} 73$ (2.65 AU at

\footnotetext{
${ }^{1}$ Columbia Astrophysics Laboratory, Mail Code 5247, 550 West 120th Street, New York, NY 10027; audard@astro.columbia.edu.

${ }^{2}$ Center for Astrophysics and Space Astronomy, University of Colorado, Boulder, CO 80309-0389; ab@ casa.colorado.edu.

${ }^{3}$ Paul Scherrer Institut, 5232 Villigen PSI, Switzerland; briggs@ astro.phys.ethz.ch, guedel@astro.phys.ethz.ch, atellesc@astro.phys.ethz.ch.

${ }^{4}$ Department of Physics and Astronomy, 223 Sharp Lab, University of Delaware, Newark, DE 19716; gizis@udel.edu.
}

a distance of 3.626 pc; Scholz et al. 2003; Smith et al. 2003; Volk et al. 2003; McCaughrean et al. 2004). We list the binary's main characteristics in Table 1. Blank (2004) failed to detect $\epsilon$ Ind Bab with ATCA, but our radio observation goes deeper.

\section{OBSERVATIONS AND DATA REDUCTION}

Despite our best effort to coordinate the Chandra and ATCA observations of the $\epsilon$ Ind Bab binary, the X-ray observations were delayed by a few days for satellite safety reasons. A log of the observations is given in Table 2.

\subsection{ATCA}

The ATCA was in a long-baseline configuration (6D); we used $4.8 \mathrm{GHz}$ and $8.64 \mathrm{GHz}$ receivers with bandwidths of $128 \mathrm{MHz}$. Observing scans ranged from 10 to 20 minutes on-source, depending on the weather conditions, whereas we used 3 minute scans for the phase calibrator. About 5 minutes at the start of each observing round were spent on the flux calibrator. The observing conditions on the first day were average, with cloud coverage; however, the last $3 \mathrm{hr}$ of the first day of observation were essentially useless as a result of strong winds and a thunderstorm. In contrast, the weather conditions were much better during the second day, with generally a cloud-free sky.

We combined both observing rounds and reduced the ATCA data using the MIRIAD software (Sault et al. 1995). We detected nine sources in the $4.8 \mathrm{GHz}$ map; we used boxes of about $20^{\prime \prime}$ width centered on these sources and applied a CLEAN algorithm using uniform weighting. About 5.4 mJy were thus removed in 202 iterations (we stopped when a negative value was encountered). Although no sources were visible in the dirty map at $8.64 \mathrm{GHz}$, we used the boxes around the first five brightest sources detected at longer wavelengths and performed a CLEAN algorithm. About $0.5 \mathrm{mJy}$ were removed after 38 iterations. The rms noise level in the cleaned maps reached 26.4 and $37.3 \mu \mathrm{Jy}$ at 4.8 and $8.64 \mathrm{GHz}$, respectively. The values are close to the theoretical values ( 25.1 and $35.6 \mu \mathrm{Jy})$. No source was detected at the expected position of the T-type brown dwarf binary (see Table 2 and Fig. 1). Since short-duration flares could be missed in the 2 day images, we have generated maps down to $1 \mathrm{hr}$ duration but still detected no source (typical rms noise levels of 120 and $155 \mu \mathrm{Jy}$ at 4.8 and $8.64 \mathrm{GHz}$, respectively). Our nondetection limits are consistent with those of Blank (2004), but 
TABLE 1

The $\epsilon$ INDI Bab BINARY

\begin{tabular}{lcc}
\hline \hline Parameter & $\epsilon$ Ind Ba & $\epsilon$ Ind Bb \\
\hline Spectral type $\ldots \ldots .$. & T1 & T6 \\
$\log \left(L_{\text {bol }} / L_{\odot}\right) \ldots \ldots$. & -4.71 & -5.35 \\
$T_{\text {eff }}(\mathrm{K}) \ldots \ldots \ldots \ldots$ & $1276 \pm 35$ & $854 \pm 20$ \\
Mass $\left(M_{\text {Jup }}\right) \ldots \ldots \ldots$ & $47 \pm 10$ & $28 \pm 7$ \\
Radius $\left(R_{\odot}\right) \ldots \ldots$. & $0.091 \pm 0.005$ & $0.096 \pm 0.005$ \\
Distance $($ pc $) \ldots \ldots$. & \multicolumn{2}{c}{3.626} \\
Age $(\mathrm{Gyr}) \ldots \ldots \ldots$ & \multicolumn{3}{c}{$0.8-2$} \\
\hline
\end{tabular}

REFERENCE. - See McCaughrean et al. (2004).

we went deeper since we observed $\epsilon$ Ind Bab about twice as long. At the distance of the binary $(3.626 \mathrm{pc})$, the $3 \sigma$ upper limits correspond to radio luminosities, $L_{R}$, of $1.23 \times 10^{12}$ and $1.74 \times 10^{12} \mathrm{ergs} \mathrm{s}^{-1} \mathrm{~Hz}^{-1}$ at 4.8 and $8.64 \mathrm{GHz}$, respectively.

\subsection{Chandra}

Chandra observed $\epsilon$ Ind Bab at two different times. We reduced both data sets with the CIAO 3.1 software in combination with CALDB 2.29. The data were taken in very faint mode, which allowed us to further reduce the background level. We later merged both event files into one single event file (total exposure of $62.5 \mathrm{ks}$ ). No signal was detected at the expected position of $\epsilon$ Ind Bab (Table 2); this was confirmed with several source detection algorithms. Figure 2 shows an extract of the Chandra ACIS-S image (0.2-8 keV). Blank (2004) tentatively associated the ROSAT source 1WGA J2203.9-5647 with $\epsilon$ Ind Bab. However, thanks the high spatial resolution of Chandra, we assign the ROSAT source to a nearby bright $\mathrm{X}$-ray source instead (see Fig. 2). No optical or near-infrared counterpart to this source could be found in Digitized Sky Survey or Two Micron All Sky Survey images.

No events were detected within 1".4 (95\% of the encircled energy at $0.3 \mathrm{keV}$ ) of the expected position of $\epsilon$ Ind Bab in the $0.2-8 \mathrm{keV}$ range. Note that, without the energy filtering, four events were detected, but all had energies above $10 \mathrm{keV}$, casting severe doubt that they were due to the source rather than the background. Furthermore, increasing the extraction radius to $3^{\prime \prime}$ only allows the detection of a single event. To estimate the background contribution, we used a concentric annulus with an inner radius of $3^{\prime \prime}$ and an outer radius calculated such that the annulus's area was 100 times larger than the area

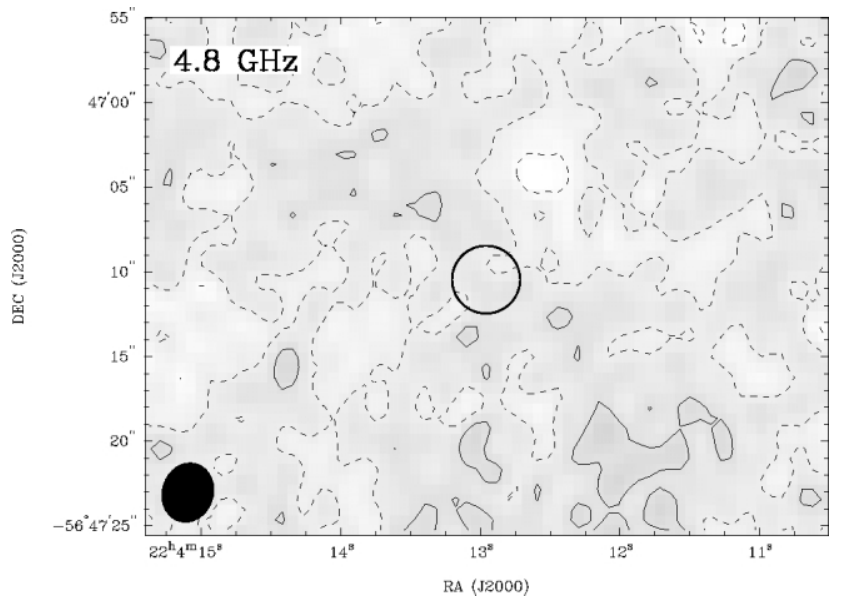

TABLE 2

OBSERVATION LOG FOR ATCA AND CHANDRA

\begin{tabular}{|c|c|}
\hline Parameter & Value \\
\hline \multicolumn{2}{|c|}{ Position of $\epsilon$ Ind Bab (Equinox: J2000; Epoch: J2004.93) } \\
\hline $\begin{array}{l}\text { Right ascension } \ldots \ldots \ldots \ldots \ldots \ldots \ldots \\
\text { Declination } \ldots \ldots \ldots \ldots \ldots \ldots \ldots \ldots\end{array}$ & $\begin{array}{l}22^{\mathrm{h}} 04^{\mathrm{m}} 12 \mathrm{~s} .96 \\
-56^{\circ} 47^{\prime} 10^{\prime \prime} .5\end{array}$ \\
\hline \multicolumn{2}{|c|}{ ATCA (Program C1269) } \\
\hline Observation date . & $\begin{array}{l}2004 \text { Dec } 4 \text { 00:30-14:30 UT } \\
2004 \text { Dec } 5 \text { 01:00-13:00 UT }\end{array}$ \\
\hline Antenna configuration & $6 \mathrm{D}$ \\
\hline Bandwidth $\ldots \ldots \ldots \ldots \ldots \ldots \ldots$ & $128 \mathrm{MHz}$ over 32 channels \\
\hline Flux calibrator $\ldots \ldots \ldots \ldots \ldots \ldots$ & PKS J1939-6342 \\
\hline (ATCA calibrator name) . & $(1934-638)$ \\
\hline Phase calibrator .......... & PMN J2121-6111 \\
\hline $\begin{array}{l}\text { (ATCA calibrator name) . } \\
8.64 \mathrm{GHz} \text { : }\end{array}$ & $(2117-61)$ \\
\hline Synthesized beam .... & $1 " .90 \times 1 " .65$ \\
\hline Position angle .... & -18.7 \\
\hline Theoretical RMS flux . & $35.56 \mu \mathrm{Jy}$ \\
\hline $4.80 \mathrm{GHz}:$ & \\
\hline Synthesized beam & 3".55 × 3.01 \\
\hline Position angle $\ldots . . .$. & -16.7 \\
\hline Theoretical RMS flux & $25.09 \mu \mathrm{Jy}$ \\
\hline \multicolumn{2}{|c|}{ Chandra (ObsID 6171 and 4485) } \\
\hline Observation date.. & $\begin{array}{l}2004 \text { Dec } 7 \text { 13:59-21:11 UT } \\
2004 \text { Dec } 900: 04-11: 35 \text { UT }\end{array}$ \\
\hline Instrument . & ACIS-S \\
\hline Total exposure & $62.4 \mathrm{ks}$ \\
\hline PSF (FWHM) $\ldots \ldots \ldots \ldots$ & 0.5 \\
\hline $95 \%$ EE PSF at $0.3 \mathrm{keV}$ & 1".44 \\
\hline
\end{tabular}

of the circle around the binary (i.e., $r_{\text {out }}=14$ "'3). A total of 77 events were detected; consequently, the scaled estimated background contribution is 0.77 events, that is, a mean background rate of 0.012 counts ks ${ }^{-1}$. We then followed the approach of Kraft et al. (1991) to determine the upper confidence limit using a Bayesian confidence level of $95 \%$, which corresponded to 2.99 counts. To convert this count rate of 0.048 counts ks ${ }^{-1}$ into an X-ray luminosity, we used one plasma component with solar photospheric abundances (Grevesse \& Sauval 1998) using the APEC 1.3.1 code (Smith et al. 2001) in the XSPEC software (Arnaud 1996). We obtained count rates in the $0.2-8 \mathrm{keV}$ band and X-ray fluxes in the $0.1-10 \mathrm{keV}$ band. The latter fluxes were converted into $\mathrm{X}$-ray luminosities, $L_{\mathrm{X}}$, using the distance of $\epsilon$ Ind Bab. For plasma temperatures of $0.4-1.0 \mathrm{keV}$, the

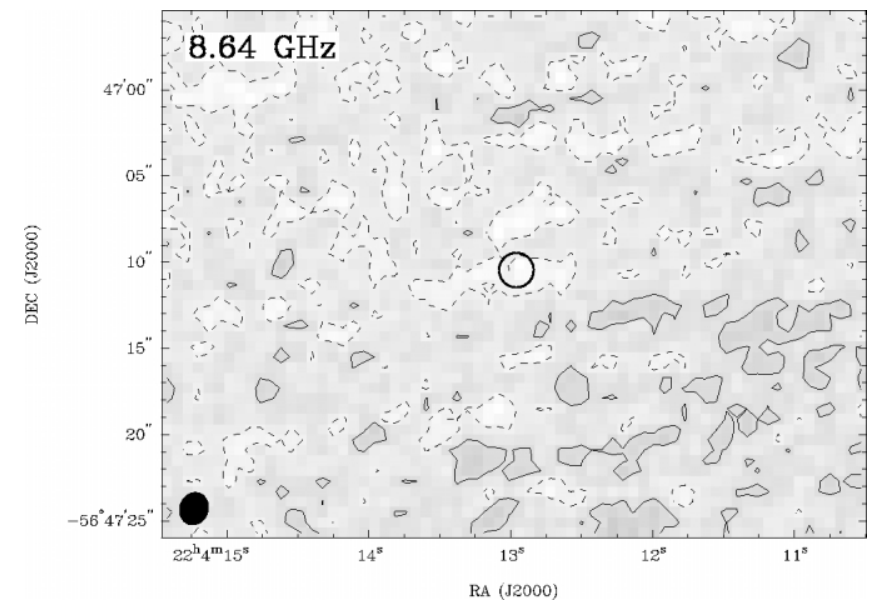

FIG. 1.-Cleaned ATCA maps of the $\epsilon$ Ind Bab field at $4.8 \mathrm{GHz}$ (left) and $8.64 \mathrm{GHz}$ (right). Contours are plotted in units of $-3,-1,1,3$, and 10 times 26.4 and $37.3 \mu \mathrm{Jy}$, respectively. The expected position of the brown dwarf binary (Table 2) is shown by open circles of $2^{\prime \prime}$ and 1 " radii, respectively. The beam size is also shown at the bottom left of each map. 


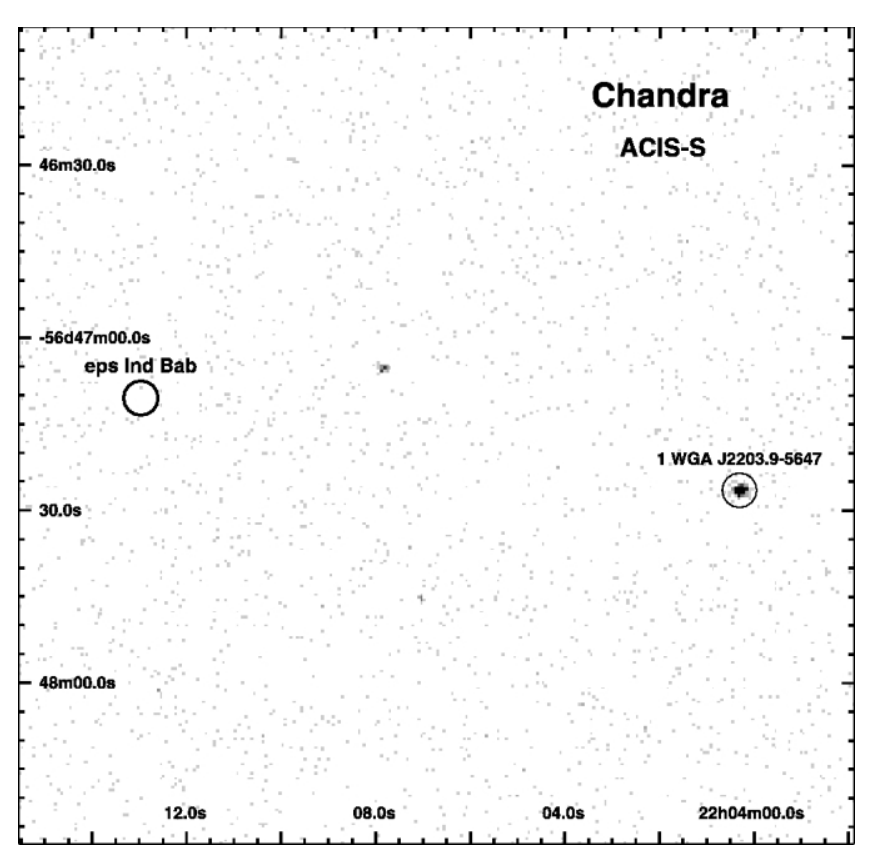

FIG. 2.-Extract of the Chandra ACIS-S image. The expected position of $\epsilon$ Ind Bab (Table 2) is shown with a circle of $3^{\prime \prime}$ radius.

conversion factor is constant, i.e., $6.5 \times 10^{24} \mathrm{ergs} \mathrm{s}^{-1}$ per count $\mathrm{ks}^{-1}$. For cooler plasma temperatures, the conversion factor increases by factors of $1.16,1.46,1.74$, and 3.25 for $k T=$ $0.3,0.2,0.15$, and $0.1 \mathrm{keV}$, respectively. Similarly, the factor increases by $1.20,1.33,1.53$, and 1.82 for $k T=1.5,2,3$, and $5 \mathrm{keV}$, respectively. Therefore, assuming $k T=0.4-1.0 \mathrm{keV}$, we obtain $L_{\mathrm{X}} \lesssim 3.16 \times 10^{23} \mathrm{ergs} \mathrm{s}^{-1}$. In the worst case $(k T=$ $0.1 \mathrm{keV})$, our upper limit increases to $1.0 \times 10^{24} \mathrm{ergs} \mathrm{s}^{-1}$.

\section{DISCUSSION}

Since ATCA could not separate the binary and Chandra would have barely done so, our upper limits $\left(L_{R}\right.$ and $\left.L_{\mathrm{X}}\right)$ can either be attributed to one binary component or the other. Table 3 gives the upper limits of the luminosity ratios to the bolometric luminosity for each component.

Our radio upper limits, $L_{R} / L_{\mathrm{bol}}<10^{-16.8}$ to $10^{-16.0} \mathrm{~Hz}^{-1}$, do not go as deep as for $\mathrm{dMe}$ stars, which are typically detected with ratios on the order of $10^{-18} \mathrm{~Hz}^{-1}$. However, the limit at $8.64 \mathrm{GHz}$ is deeper for $\epsilon$ Ind $\mathrm{Ba}$ than the upper limit obtained at $8.46 \mathrm{GHz}$ with the VLA by Berger (2002) for a T6 brown dwarf (our limit at $8.64 \mathrm{GHz}$ for $\epsilon$ Ind $\mathrm{Bb}$ is, however, equivalent). Berger hypothesized that the radio luminosity relative to the bolometric luminosity would increase with later spectral type, and their strong detection of an L dwarf in radio appears to support this (Berger et al. 2005). Similarly, despite the lack of sensitivity of current radio instruments, such a trend is also suggested in a small survey of southern late $\mathrm{M}$ and $\mathrm{L}$ dwarfs (Burgasser \& Putman 2005). On the other hand, our upper limits for $\mathrm{T}$ dwarfs do not indicate that this trend continues into the $\mathrm{T}$ dwarf domain. Deep observations of $\mathrm{T}$ dwarfs can, in principle, be very useful, since they provide data points at low $T_{\text {eff }}$; however, the exposure required to achieve the necessary sensitivity for comparison with dMe dwarfs (i.e., $L_{R} /$ $L_{\text {bol }}<10^{-18}$ to $10^{-19} \mathrm{~Hz}^{-1}$ ) must await the advent of the next generation of radio telescopes.

In contrast, the sensitivity of Chandra allows us to obtain a low $L_{\mathrm{X}} / L_{\text {bol }}$ ratio. We reach an upper limit about $10-100$ times lower than the $L_{\mathrm{X}} / L_{\text {bol }}$ ratios observed in active dMe stars (e.g.,
TABLE 3

UPPER LIMITS OF LUMINOSITIES AND OF LUMINOSITY RATIOS TO THE BOLOMETRIC LUMINOSITY

\begin{tabular}{crc}
\hline \hline Upper Limit & $\epsilon$ Ind Ba & $\epsilon$ Ind Bb \\
\hline$L_{R, 4.8 \mathrm{GHz}}\left(\mathrm{ergs} \mathrm{s}^{-1} \mathrm{~Hz}^{-1}\right) \ldots \ldots \ldots$ & $1.23 \times 10^{12}$ \\
$L_{R, 8.64 \mathrm{GHz}}\left(\operatorname{ergs~s}^{-1} \mathrm{~Hz}^{-1}\right) \ldots \ldots \ldots$ & $1.74 \times 10^{12}$ \\
$L_{\mathrm{X}, 0.1-10 \mathrm{keV}}\left(\mathrm{ergs} \mathrm{s}^{-1}\right) \ldots \ldots \ldots \ldots$ & $3.16 \times 10^{23}$ \\
$\log \left(L_{R, 4.8 \mathrm{GHz}} / L_{\mathrm{bol}}\right)\left(\mathrm{Hz}^{-1}\right) \ldots \ldots \ldots$ & $-16.79^{\mathrm{a}}$ & $-16.15^{\mathrm{a}}$ \\
$\log \left(L_{R, 8.64 \mathrm{Gz}} / L_{\mathrm{bol}}\right)\left(\mathrm{Hz}^{-1}\right) \ldots \ldots \ldots$ & $-16.64^{\mathrm{a}}$ & $-16.00^{\mathrm{a}}$ \\
$\log \left(L_{\mathrm{X}, 0.1-10 \mathrm{kev}} / L_{\mathrm{bol}}\right) \ldots \ldots \ldots \ldots \ldots$ & $-5.38^{\mathrm{a}}$ & $-4.74^{\mathrm{a}}$ \\
\hline
\end{tabular}

${ }^{a}$ Ratios calculated by attributing the luminosity upper limits to either component.

Fleming et al. 1995, 2003). In fact, our limit is close to the ratio observed in the Sun (as a star) at its activity maximum and to the ratio observed in early $\mathrm{dM}$ stars that do not show $\mathrm{H} \alpha$ in emission (Fleming et al. 1995). Although X-ray observations of ultracool dwarfs are rare (e.g., Rutledge et al. 2000; Schmitt \& Liefke 2002; Martín \& Bouy 2002; Fleming et al. 2003; Briggs \& Pye 2004; Stelzer 2004; Berger et al. 2005), the X-ray emission in such dwarfs appears to decline like the $\mathrm{H} \alpha$ emission. Most X-ray detections were actually obtained during flares, with only a few objects detected in quiescence. Our nondetection of the T-type $\epsilon$ Ind Bab with Chandra reinforces the view that the X-ray emission in ultracool dwarfs declines significantly with later spectral type.

Berger et al. (2005) suggested that magnetic activity is strong in ultracool dwarfs, in fact much stronger than in dMe stars, but that it manifests itself mostly in the radio, as atmospheric conditions in such dwarfs become unfavorable for $\mathrm{H} \alpha$ and $\mathrm{X}$ ray emission because of the decoupling of the magnetic field from the neutral photospheric gas (Meyer \& Meyer-Hofmeister 1999; Mohanty et al. 2002). Taken alone, the lack of X-rays from $\epsilon$ Ind Bab could, in principle, support this view. However, Berger (2002) noted that fast rotators $\left(>10 \mathrm{~km} \mathrm{~s}^{-1}\right)$ have high $L_{R} / L_{\text {bol }}$ ratios. But $\epsilon$ Ind Bab does not follow this trend: Smith et al. (2003) found that the T1 component is a fast rotator ( $v$ $\left.\sin i=28 \mathrm{~km} \mathrm{~s}^{-1}\right)$. Note that the stellar radius and period are similar to those of the L3.5 dwarf detected in radio (but not in X-rays or $\mathrm{H} \alpha$ ) by Berger et al. (2005). Possibly, the suggested trend observed in late M/early L dwarfs does not hold in the old, cool T dwarfs ( $\left.T_{\text {eff }}=800-1300 \mathrm{~K}\right)$. Our observation thus suggests a physical change in magnetic behavior somewhere in the range of $\mathrm{T}$ dwarfs. Alternatively, the observed sample of $\mathrm{L}$ and $\mathrm{T}$ dwarfs is too limited and sensitivity is lacking.

Magnetically active stars often show a relation between the quiescent X-ray and radio luminosities (Güdel \& Benz 1993). The Güdel-Benz relation points toward a close connection between nonthermal electrons observed as gyrosynchrotron emission and the thermal plasma radiating in X-rays. The origin of this connection remains unclear; however, there is increasing evidence that the X-ray emission in magnetically active stars is due to the statistical superposition of flares (e.g., Audard et al. 2000; Güdel et al. 2003). In addition, the radio and X-ray light curves of flares are often correlated (e.g., Neupert 1968; Güdel et al. 2002). Thus, coronal heating by flares provides an elegant explanation for the time-average $L_{\mathrm{X}}-L_{R}$ relation observed in active stars. However, ultracool dwarfs seem not to follow this relation, since they show stronger radio emission with respect to the X-ray emission (e.g., Berger et al. 2001; Berger 2002; Burgasser \& Putman 2005). This indicates either that magnetic activity in such dwarfs is different (Berger et al. 2001, 2005), or possibly that the radio emission mechanism differs significantly from gyrosynchrotron. Indeed, the emission 
of many flares in $\mathrm{M}$ dwarfs can be ascribed to a coherent mechanism (e.g., Kundu et al. 1988). In the case of $\epsilon$ Ind Bab, we obtained only upper limits for both radio and X-ray regimes; thus, it is unclear whether the T brown dwarfs follow the GüdelBenz relation or not.

\section{CONCLUSIONS}

We have presented deep observations of $\epsilon$ Ind Bab, a T1 + T6 brown dwarf binary at $3.626 \mathrm{pc}$, in X-rays with Chandra and in the radio with ATCA. The binary remained undetected, with upper limits of $L_{\mathrm{X}} / L_{\mathrm{bol}} \lesssim 10^{-5.4}$ and $10^{-4.7}$ and $L_{R} / L_{\mathrm{bol}} \lesssim 10^{-16.8}$ and $10^{-16.2} \mathrm{~Hz}^{-1}$ (at $4.8 \mathrm{GHz}$ ) for $\epsilon$ Ind $\mathrm{Ba}$ and $\mathrm{Bb}$, respectively. The nondetection in the radio is in contrast with the trend of increasing $L_{R} / L_{\text {bol }}$ with later spectral type suggested by Berger (2002) and Berger et al. (2005) in late $\mathbf{M}$ and L dwarfs. Berger et al. (2005) argued that the neutral atmospheres in ultracool dwarfs lead to unfavorable conditions for the X-ray and $\mathrm{H} \alpha$ emissions (Meyer \& Meyer-Hofmeister 1999; Mohanty et al. 2002), but not for the radio emission of fast rotators. However, the $\mathrm{T} 1$ component in $\epsilon$ Ind $\mathrm{Bab}$ is a rapid rotator with similar period and radius as the $\mathrm{L}$ dwarf detected strongly in the radio (but undetected in X-rays and $\mathrm{H} \alpha$ ) by Berger et al. (2005). Therefore, our $\epsilon$ Ind Bab observations indicate that cool $\mathrm{T}$ dwarfs may not follow the trend suggested by Berger and colleagues. However, a definitive conclusion is difficult to reach in view of the limited sample of ultracool dwarfs observed in the radio and in X-rays and of the lack of sensitivity of present radio (and $\mathrm{X}$-ray) instruments.

The Australia Telescope Compact Array is part of the Australia Telescope, which is funded by the Commonwealth of Australia for operation as a National Facility managed by CSIRO. Support for this work was provided by the National Aeronautics and Space Administration through Chandra award G04-5002X to Columbia University and issued by the Chandra $X$-Ray Observatory Center, which is operated by the Smithsonian Astrophysical Observatory for and on behalf of the NASA under contract NAS8-03060, and by National Science Foundation grant AST 02-06367 and NASA grant NAG 513058 to the University of Colorado. The PSI group acknowledges support from the Swiss National Science Foundation (grants 20-58827.99 and 20-66875.01). We thank two referees, Mark Giampapa and an anonymous one, for useful comments and suggestions that improved the content of this paper. M. A. thanks Scott Wolk and Bob Sault for their efforts to coordinate the Chandra and ATCA observations.

\section{REFERENCES}

Arnaud, K. A. 1996, in ASP Conf. Ser. 101, Astronomical Data Analysis Software and Systems V, ed. G. H. Jacoby \& J. Barnes (San Francisco: ASP), 1

Audard, M., Güdel, M., Drake, J. J., \& Kashyap, V. L. 2000, ApJ, 541, 396 Berger, E. 2002, ApJ, 572, 503

Berger, E., et al. 2001, Nature, 410, 338

. 2005, ApJ, in press (astro-ph/0502384)

Blank, D. L. 2004, MNRAS, 354, 913

Briggs, K. R., \& Pye, J. P. 2004, MNRAS, 353, 673

Burgasser, A. J., \& Putman, M. E. 2005, ApJ, in press (astro-ph/0502365)

Drake, J. J., Stern, R. A., Stringfellow, G. S., Mathioudakis, M., Laming, J. M., \& Lambert, D. L. 1996, ApJ, 469, 828

Durney, B. R., De Young, D. S., \& Roxburgh, I. W. 1993, Sol. Phys., 145, 207

Fleming, T. A., Giampapa, M. S., \& Garza, D. 2003, ApJ, 594, 982

Fleming, T. A., Giampapa, M. S., \& Schmitt, J. H. M. M. 2000, ApJ, 533, 372

Fleming, T. A., Giampapa, M. S., Schmitt, J. H. M. M., \& Bookbinder, J. A. 1993, ApJ, 410, 387

Fleming, T. A., Schmitt, J. H. M. M., \& Giampapa, M. S. 1995, ApJ, 450, 401

Gizis, J. E., Monet, D. G., Reid, I. N., Kirkpatrick, J. D., Liebert, J., \& Williams, R. J. 2000, AJ, 120, 1085

Grevesse, N., \& Sauval, A. J. 1998, Space Sci. Rev., 85, 161

Güdel, M., Audard, M., Kashyap, V. L., Drake, J. J., \& Guinan, E. F. 2003, ApJ, 582, 423

Güdel, M., Audard, M., Skinner, S. L., \& Horvath, M. I. 2002, ApJ, 580, L73
Güdel, M., \& Benz, A. O. 1993, ApJ, 405, L63

Johnson, H. M. 1981, ApJ, 243, 234

1987, ApJ, 316, 458

Kraft, R. P., Burrows, D. N., \& Nousek, J. A. 1991, ApJ, 374, 344

Kundu, M. R., Pallavicini, R., White, S. M., \& Jackson, P. D. 1988, A\&A, 195,159

Martín, E. L., \& Bouy, H. 2002, NewA, 7, 595

McCaughrean, M. J., Close, L. M., Scholz, R.-D., Lenzen, R., Biller, B., Brandner, W., Hartung, M., \& Lodieu, N. 2004, A\&A, 413, 1029

Meyer, F., \& Meyer-Hofmeister, E. 1999, A\&A, 341, L23

Mohanty, S., \& Basri, G. 2003, ApJ, 583, 451

Mohanty, S., Basri, G., Shu, F., Allard, F., \& Chabrier, G. 2002, ApJ, 571, 469

Neupert, W. M. 1968, ApJ, 153, L59

Rutledge, R. E., Basri, G., Martín, E. L., \& Bildsten, L. 2000, ApJ, 538, L141

Sault, R. J., Teuben, P. J., \& Wright, M. C. H. 1995, in ASP Conf. Ser. 77, Astronomical Data Analysis Software and Systems IV, ed. R. A. Shaw, H. E. Payne, \& J. J. E. Hayes (San Francisco: ASP), 433

Schmitt, J. H. M. M., \& Liefke, C. 2002, A\&A, 382, L9

Scholz, R.-D., McCaughrean, M. J., Lodieu, N., \& Kuhlbrodt, B. 2003, A\&A, 398, L29

Smith, R. K., Brickhouse, N. S., Liedahl, D. A., \& Raymond, J. C. 2001, ApJ, 556, L91

Smith, V. V., et al. 2003, ApJ, 599, L107

Stelzer, B. 2004, ApJ, 615, L153

Tagliaferri, G., Doyle, J. G., \& Giommi, P. 1990, A\&A, 231, 131

Volk, K., Blum, R., Walker, G., \& Puxley, P. 2003, IAU Circ. 8188 